\section{Autoimmune thyroid disease: What secrets we still need to unravel?}

José Augusto Sgarbi'

A utoimmune thyroid disease (AITD) has been considered the prototypical and the most prevalent organ-specific autoimmune disorder, affecting $2 \%$ to $5 \%$ of iodine-sufficient population (1). This condition encompasses a clinical-pathological spectrum of different phenotypes varying from hyperthyroidism in Graves' disease to hypothyroidism in Hashimoto's thyroiditis, probably representing the net effect of a complex mechanism causing break of tolerance to thyroid proteins through interaction between genetic susceptibility and environmental triggers (2-4). The rational for this theory is based on both family studies and in twins based studies showing respectively a higher frequency of AITD in the relatives of an affected individual and a higher but not complete concordance among identical twins (5).

Several genes have been associated with AITD, including immune-response genes (HLADRA II and CD40), T-cell regulation genes (CTLA4, PTPN22, CD25), and thyroid-specific antigens genes (TG, and TSHR). However, these genes together probably do not confer more than about $10 \%$ of the genetic susceptibility for AITD (6). In other words, there is no single or dominant gene strong enough to confer major susceptibility to AITD. Thus, the disease seems likely to result from the combined effect of multiple genes (2-6).

The most important environmental factors that have been implicated with AITD include iodine intake, stress, smoking, selenium, drugs, and infections (6). However, the mechanisms by which environmental factor trigger thyroid autoimmunity in genetically primed individuals remain uncertain.

This current issue of Archives of Endocrinology and Metabolism contains two articles conducted in the city of Rio de Janeiro that address different aspects of AITD: one study (7) evaluated the frequency of thyroid diseases in first degree relatives (FDR) of patients with type 1 diabetes mellitus (TIDM), and the other analyzed the prevalence of thyroid autoimmunity in HIV-infected patients (8). Araujo and cols. (7) found a high and similar prevalence of thyroid dysfunction and autoimmunity in patients with TIDM and their FDR. These data reinforce the theory of familial autoimmunity, which occurs when relatives from an affected individual present diverse autoimmunity diseases $(9,10)$. Indeed, a strong genetic association between TIDM and AITD has been demonstrated, indicating but not proving that TIDM and AITD share similar genetic susceptibility genes $(11,12)$. Identifying how do autoimmune diseases cluster in families and the basis for this shared pathogenesis is important to better understanding the mechanisms of autoimmune diseases and the origin of AITD. In addition, identification of specific genetic markers associated with AITD could improve our ability to predict autoimmunity, disease progression, and their morbidities (10). It could be of particular interest on screening and prevention strategies for subclinical hypothyroidism due to potential associations between such a condition with a higher cardiovascular risk and mortality (13). However, to date only determination of thyroid
${ }^{1}$ Division of Endocrinology and Metabolism, Department of Medicine, Faculdade de Medicina de Marília (Famema) Marília, SP, Brazil

Correspondence to: José Augusto Sgarbi

Divisão de Endocrinologia e Metabolismo, Faculdade de Medicina de Marília Av. Tiradentes, 1310 17519-000 - Marília, SP, Brazil jasgarbi@famema.br

Received on Mar/31/2015 Accepted on Mar/31/2015

DOI: 10.1590/2359-3997000000019 
antibodies has been used in practice to differentiate clinical phenotypes and disease progression of AITD.

The results found by Araujo and cols. (7) may also have implications to the clinical practice by suggesting that an extending screening for AITD would also be beneficial to FDR of TIDM patients. However, some caution in potential implications drawn from these results is required due to important methodological limitations, such as its cross-section design and the modest sample size. Thus, further larger prospective studies are necessary to clarify the strength of the association between TIDM and AITD, and also to verify whether a universal screening for AITD and thyroid dysfunctions should be recommended for FDR of affected individuals.

In the study by Rosa da Silva and cols., (8) a lower prevalence $(4 \%)$ for positive anti-thyroperoxidase antibodies (TPO-Ab) was found among $117 \mathrm{HIV}$-infected patients compared to the expected for the general population (14), but it is in line with a previous published study in the same population (15). This result is surprisingly considering the high prevalence $(12.8 \%)$ of subclinical hypothyroidism found in this sample, and suggests an environmental protective factor in HIV-infected patients to thyroid autoimmunity. In fact, a previous study pointed that subclinical hypothyroidism in HIV-infected patients is not an autoimmune disease (16).

It has been postulated that nearly $80 \%$ of the susceptibility to develop AITD is attributed to genetic factors, while environmental factors contribute to nearly $20 \%$ (5). In this model of disease development, environmental factor is essential for trigger thyroid autoimmunity, but mechanisms of interaction with susceptibility genes are not fully understood. Most recently, new players has been added in the knowledge aiming to decipher the secrets of interaction risk factors. It has been hypothesized that non-infectious environmental stress could reactivate viral latent infections and triggering an autoimmune disease in genetically primed individuals (17). In addition, epigenetic modulation factors have been implicated as a plausible mechanism for interaction between susceptibility genes and environmental triggers. Interpheron alpha (IFN- $\alpha$ ), a cytokine secreted by viruses infections, was shown to induce alterations in thyroglobulin gene expression through epigenetic modifications in histone $(3,4)$. Taken together, these recent players highlights to a multifactorial mechanism in which non-infections risk factors (stress), infectious risk factors (viruses), epigenetic factors (IFN- $\alpha$ ), and susceptibility genes (TG) act in concert to cause AITD. Furthermore, a moderate alcohol intake and smoking have been associated with a decreased risk for Hashimoto's thyroiditis (6). The female preponderance for AITD might now be associated to fetal microchimerism despite epidemiological studies are controversial on this matter (18). Interestingly, the use of antiretrovirals has been associated with high frequency of subclinical hypothyroidism, but not associated with $\operatorname{AITD}(16)$. Unfortunately, there is no date about alcohol consumption and smoking in the study by Rosa da Silva and cols. (8), being impossible to estimate potential influences of these modifiable environmental factors on their results, and whether use of antiretrovirals could affect or modulate some epigenetic factor involved in the interaction between susceptibility genes and environmental factors is unknown.

Despite considerable progress in understanding the mechanisms involved in the pathogenesis of AITD, many secrets still need to be unravel. A continuous effort by researchers is yet necessary to identify novel and stronger genes of susceptibility and by which exact mechanisms they interact with environmental factors and epigenetic factors to cause AITD. Identifying specific genetic markers and modifiable environmental factors associated to AITD, understanding how do AITD cluster in families, what is the basis for a shared pathogenesis with other autoimmune diseases, and how to explain the female preponderance will certainly provide new insights in the knowledge about the origin of AITD, as well as will probably improve our ability to predict AITD and avoid progression to a more advanced disease and its complications.

Disclosure: no potential conflict of interest relevant to this article was reported.

\section{REFERENCES}

1. Sgarbi JA, Maciel RM. Pathogenesis of autoimmune thyroid diseases. Arq Bras Endocrinol Metabol. 2009;53:5-14.

2. McLachlan SM, Rapoport B. Breaking tolerance to thyroid antigens: changing concepts in thyroid autoimmunity. Endocr Rev. 2014;35:59-105.

3. Hasham A, Tomer Y. Genetic and epigenetic mechanisms in thyroid autoimmunity. Immunol Res. 2012;54:204-13.

4. TomerY. Mechanisms of autoimmune thyroid diseases: from genetics to epigenetics. Annu Rev Pathol. 2014;9:147-56.

5. BrixTH, Kyvik KO, Chridtensen K, Hegedus L. Evidence for a major role of heredity in Graves' disease: a population-based study of two Danish twin cohorts. J Clin Endocrinol Metab. 2001;86:930-4. 
6. Effraimidis G, Wiersinga WM. Mechanisms in endocrinology: autoimmune thyroid disease: old and new players. Eur $\mathrm{J}$ Endocrinol. 2014;170:R241-52.

7. Araujo DB, Barone B, Melleti NFT, Oliveira MMS, Zadjenverg L, Tortora RP, et al. Thyroid disorders are common in first-degree relatives of individuals with type 1 diabetes mellitus. Arch Endocrinol Metab. 2015;59(2):112-5.

8. Rosa da Silva GA, Andrade MC, Sugui DA, Nunes RF, Cunha Pinto $\mathrm{JF}$, Silva WA, et al. Association between antiretrovirals and thyroid disease: a cross-sectional study. Arch Endocrinol Metab. 2015;59(2):116-22.

9. Weetman AP. Diseases associated with thyroid autoimmunity: explanations for the expanding spectrum. Clin Endocrinol (Oxf). 2011;74: 411-8.

10. Cárdenas-Roldán J, Rojas-Villarraga A, Anaya JM. How do autoimmune diseases cluster in families? A systematic review and meta-analysis. BMC Med. 2013;11:73.

11. TomerY, Menconi F.Type 1 diabetes and autoimmune thyroiditis: the genetic connection. Thyroid. 2009;19:99-102.

12. Hansen MP, Matheis N, Kahaly HJ.Type 1 diabetes and polyglandular autoimmune syndrome: a review. World J Diabetes. 2015;6:67-79.
13. Sgarbi JA, Matsumura LK, KasamatsuTS, Ferreira SR, Maciel RM. Subclinical thyroid dysfunctions are independent risk factors for mortality in a 7.5-year follow-up: the Japanese-Brazilian thyroid study. Eur J Endocrinol. 2010;162:569-77.

14. Sichieri R, Baima J, Marante T, de Vasconcellos MT, Moura AS, Vaisman M. Low prevalence of hypothyroidism among black and Mulatto people in a population-based study of Brazilian women. Clin Endocrinol (Oxf). 2007;66:803-7.

15. Carvalho LG, Teixeira Pde F, Panico AL, Cohen MV, Pinheiro MF, Barroso $\mathrm{PF}$, et al. Evaluation of thyroid function and autoimmunity in HIV-infected women. Arq Bras Endocrinol Metabol. 2013;57:450-6.

16. Temajo NO, Howard N. The virus-induced HSPs regulate the apoptosis of operates APCs that results in autoimmunity, not in homeostasis. Autoimmun Rev. 2014;13:1013-9.

17. Beltran S, Lescure FX, Ei Esper I, Schimit JL, Desailloud R. Subclinical hypothyroidism in HIV infected patients is not an autoimmune disease. Horm Res. 2006;66:21-6.

18. Sgarbi JA, Kasamatsu TS, Matsumura LK, Maciel RM. Parity is not related to autoimmune thyroid disease in a population-based study of Japanese-Brazilians. Thyroid. 2010;20:1151-6. 